\title{
Determination of Transport Properties From Flowing Fluid Temperature Logging In Unsaturated Fractured Rocks: Theory And Semi-Analytical Solution
}

\author{
Sumit Mukhopadhyay and Yvonne W. Tsang \\ Lawrence Berkeley National Laboratory, Berkeley, CA 94720
}

\begin{abstract}
Flowing fluid temperature logging (FFTL) has been recently proposed as a method to locate flowing fractures. We argue that FFTL, backed up by data from high-precision distributed temperature sensors, can be a useful tool in locating flowing fractures and in estimating the transport properties of unsaturated fractured rocks. We have developed the theoretical background needed to analyze data from FFTL. In this paper, we present a simplified conceptualization of FFTL in unsaturated fractured rock, and develop a semianalytical solution for spatial and temporal variations of pressure and temperature inside a borehole in response to an applied perturbation (pumping of air from the borehole). We compare the semi-analytical solution with predictions from the TOUGH2 numerical simulator. Based on the semi-analytical solution, we propose a method to estimate the permeability of the fracture continuum surrounding the borehole. Using this proposed method, we estimated the effective fracture continuum permeability of the unsaturated rock hosting the Drift Scale Test (DST) at Yucca Mountain, Nevada. Our estimate compares well with previous independent estimates for fracture permeability of the DST host rock. The conceptual model of FFTL presented in this paper is based on the assumptions of single-phase flow, convection-only heat transfer, and negligible change in system state of the rock formation. In a sequel paper [Mukhopadhyay et al., 2008], we extend the conceptual model to evaluate some of these assumptions. We also perform inverse modeling of FFTL data to estimate, in addition to permeability, other transport parameters (such as porosity and thermal conductivity) of unsaturated fractured rocks.
\end{abstract}

\section{Introduction}

Borehole temperature logs have been used in a variety of engineering and scientific applications [Jessop, 1990; Prensky, 1992]. Continuously (in time) recording borehole temperature logs, especially when recorded in boreholes under equilibrium conditions, provide useful information about the subsurface thermal structure [Förster et al., 1997]. Consequently, borehole temperature logs have been used for determination of subsurface thermal conductivity and lithology [e.g., Blackwell et al., 1999], and coupled heat and fluid flow through porous and fractured rock [Smith and Chapman, 1983; Drury et al., 1984; Ge, 1998; Constanz et al., 2003]. Borehole temperature logs have also been used in the analysis of surface features and processes such as topography, differential solar 
radiation, vegetation, and past changes in surface temperature [e.g., Chisholm and Chapman, 1992; Harris and Chapman, 1995].

The borehole-temperature-logging applications mentioned above all occur under conditions of no induced fluid flow. However, since not all the fractures intersected by a borehole contribute to the flow process, borehole logging performed under non flowing conditions is not always useful for fracture detection and property estimation. Tsang et al. [1990] introduced a flowing fluid electrical conductivity log (FFECL) for characterization of fractured reservoirs to identify fractures that contribute to flow. The FFECL has been successfully employed in characterization of various reservoirs [Tsang et al., 1990; Tsang and Doughty, 2003; Doughty and Tsang, 2005; Doughty et al., 2005].

More recently, Tsang et al. [2007] introduced flowing fluid temperature logging (FFTL) for characterizing unsaturated fractured reservoirs. They propose thermal logging along a borehole during constant-rate pumping conditions to measure the transport parameters of unsaturated fractured rock. Since the rock is unsaturated, the fluid being pumped is air (which intrinsically contains water vapor). As air enters the borehole from conducting fractures intercepted by the borehole, it undergoes a change in pressure, and consequently, a temperature signal can be measured. The depths in the borehole of the signals indicate the locations of the hydraulically conducting fractures, within a certain resolution, and its magnitude and time change would indicate the permeability and other transport properties of the fracture (see below for further elaboration).

FFTL, in our opinion, has significant potential to determine of the location of flowing fractures and the associated parameters. However, the success of FFTL will be largely controlled by the accuracy of measured temperatures. In this context, we are encouraged by the recent emergence of high-precision distributed temperature sensing systems (DTS). The DTS allows collection of temperature data continuously over the length of an entire borehole [Hurtig et al., 1993; Hurtig et al., 1994; Osato et al., 1995; Sakaguchi and Matsushima, 1995; Benoit and Thompson, 1998; Wisian et al., 1998; Sakaguchi and Matsushima, 2000; Ikeda et al., 2000; Ikeda, 2003; Henninges et al., 2005; Selker et al. 
2006]. With continued improvement in measurement precision, it may be possible to use FFTL to estimate transport properties with significant accuracy. DTS-based FFTL will also eliminate the logistical difficulty of conventional FFTL, where the logging tool has to be moved from location to location as one logs an entire borehole. In addition, DTSbased FFTL can be useful for capturing spatial heterogeneity in transport properties at different scales. In short, FFTL, backed by DTS, has a great potential to be a viable method for estimation of transport properties.

Conventionally, pressure data from boreholes have been used to estimate permeability. For example, soil scientists have routinely injected air and measured soil air pressure to evaluate soil permeability [e.g., Kirkham, 1946]. More recently, hydrologists have applied similar air-injection techniques to evaluate the permeability [Huang et al., 1999] and/or fracture porosity [Freifeld, 2001] of unsaturated fractured rock. The underlying theory supporting estimation of hydraulic properties from air-injection testing in unsaturated fractured rock is well established [see Freifeld, 2001 and references therein]. However, estimation of permeabilities from FFTL data in unsaturated rock is a relatively new approach, and no theoretical basis is currently available in the literature, though a very preliminary analysis of FFTL was presented in Tsang et al. [1994].

We have developed the necessary theoretical basis for analyzing and interpreting FFTL data from unsaturated fractured rock. We shall present the results of our study in two papers. In this paper, we present a semi-analytical solution for transient pressure and temperature signature in the borehole, assuming that only air is present, i.e., ignoring the water vapor in the air phase. This semi-analytical solution is verified against results from the numerical simulator TOUGH2 [Pruess, 1991; Pruess et al., 1999]. Next, the semianalytical solution was used to obtain a first-order estimate of permeability of the fractured rock. In a sequel to this paper (hereafter referred to as Paper II) [Mukhopadhyay et al., 2008], we extend the conceptual model to further investigate the more subtle features of measured FFTL signatures. In Paper II, we perform a systematic sensitivity study to determine which transport parameters most strongly influence measured FFTL 
signatures. Paper II also contains results of inverse numerical simulations for estimation of transport properties from FFTL data.

\section{Sample FFTL Data}

The FFTL data presented in this paper were collected using a temperature logging tool (see Figure 1 for photographs of the logging tool), the design of which can be found in Tsang et al. [2007]. Using inflatable packers, the logging tool can be used to pack off a $0.9 \mathrm{~m}$ zone of a borehole at any one time. The logging tool has five temperature sensors (linear thermisters) at a gap of about $0.15 \mathrm{~m}$ with one another. Out of the five temperature sensors, Sensor \#1 is always located at the deepest end of the logging tool and Sensor \#5 is located closest to the end where pumping is applied.

Temperature and pressure data were collected by introducing the logging tool in Borehole 182 at Alcove 5 of the Exploratory Studies Facility (ESF) at Yucca Mountain, Nevada. The ESF at Yucca Mountain was constructed to facilitate characterization of Yucca Mountain as the site for the US high-level radioactive waste repository. Alcove 5 is located in the middle nonlithophysal unit (Tptpmn) of the Topopah Spring Tuff at Yucca Mountain. Borehole 182 is $20 \mathrm{~m}$ long and has a diameter of $0.076 \mathrm{~m}$. It originates at the Observation Drift (OD) near the Drift Scale Test (DST) (the largest in situ heater test in the unsaturated fractured rock of Yucca Mountain, see, for example Birkholzer and Tsang, 2000; Mukhopadhyay and Tsang, 2003; Mukhopadhyay et al., 2007) site and is oriented at about $19^{\circ}$ with the vertical going downwards and below the Heated Drift (HD) of the DST. Previous characterization of the Tptpmn stratigraphic unit has shown the rock to be highly fractured, with the fractures forming a well-connected network [Sonnenthal et al., 1997]. The fracture permeability was found to be varying over four orders of magnitude [Huang et al., 1999; Birkholzer and Tsang, 2000]. A geometric mean permeability of $1.3 \times 10^{-13} \mathrm{~m}^{2}$ has been previously accepted [Birkholzer and Tsang, 2000] as a representative fracture continuum permeability of the Tptpmn rock. 
The logging tool was first introduced near the end (i.e., the farthest from the OD) of Borehole 182. Packers were inflated to isolate the $0.9 \mathrm{~m}$ section of the logging tool. Air was pumped at a constant flow rate for a certain amount of time (typically five to six minutes), and temperatures were recorded by the five sensors. Pressure and flow rate data were also recorded. The packers were then deflated and the logging tool was moved to a different part of Borehole 182, and the procedure (packer inflation, pumping, and data recording) was repeated. In total, about 50 such pumping and data collection cycles were completed in Borehole 182 during the day of experiment.

A typical set of recorded data during the logging experiment is shown in Figure 2, corresponding to when the logging tool was located at about $8.4 \mathrm{~m}$ from the end of the borehole (or approximately $11.6 \mathrm{~m}$ from the collar). For this particular cycle of data collection, air was pumped at an average rate of approximately 25.2 SLPM (standard liter per minute) or $5.43 \times 10^{-4} \mathrm{~kg} / \mathrm{s}$ for 322 seconds. The pump rate (or flow rate) of air and the pressure inside the borehole during this logging experiment are shown in Figure 2a. Temperatures recorded by the five sensors are shown in Figure 2b. In Figure 2b, Sensor \#5 is closest to that end of the logging tool where pumping is applied, and Sensor \#1 is the farthest (i.e., zone 1 is situated deepest into the borehole). Except for Sensor \#5, temperatures recorded by the other four sensors are similar. All of them exhibit a sharp decline in temperature at the start of pumping before gradually recovering to their original, pre-pumping values. When pumping is stopped, temperatures went above their original values. In other words, cooling was observed in the borehole at the commencement of pumping, and heating trends were observed as pumping was stopped. Contrary to the temperature history recorded by Sensors \#1 through \#4, persistent cooling trends were recorded by Sensor \#5 throughout the pumping period, and temperature began to increase only after pumping was stopped. We are yet to find an explanation for the temperature recording by Sensor \#5. One possible explanation could be that, for this particular location, the rock closest to Sensor 5 was relatively less fractured (that is why temperature dropped in response to pumping but did not rebound because no fracture or fractures were feeding into the borehole at that location - see Section 3 for more details). However, to test the above hypothesis, more analysis is 
needed. Consequently, in the rest of this paper, we will focus on temperatures recorded by Sensors \#1 through \#4. Note that not including data from Sensor \#5 does not impact the rest of the analysis presented in this paper.

\section{Interpretation of Observed Data}

When air is pumped from a borehole with fixed volume, both pressure and temperature inside the borehole decrease. This is a situation similar to an adiabatic expansion experiment. If pumping is performed slowly (resembling a reversible process in a thermodynamic sense), the drop in temperature of an ideal gas will be related to the drop in its pressure, through the adiabatic pressure-temperature relationship:

$$
\frac{T_{F}}{T_{i}}=\left(\frac{P_{F}}{P_{i}}\right)^{\frac{\gamma-1}{\gamma}}
$$

In Equation 1, $\gamma$ is the ratio of specific heat capacity of air (assumed an ideal gas) at constant pressure to that at constant volume, i.e., $\gamma=\frac{C_{P}}{C_{V}}$. Because the borehole is located in a highly fractured rock, which acts as a source of fluid (air in this case), temperature (and pressure) signature from FFTL differs from that of a reversible adiabatic expansion experiment. For example, as pressure drops inside the borehole because of pumping, air (along with water vapor) begins to flow from the fractured rock into the borehole. However, since the fractured rock has a finite permeability, it takes some time (i.e., there is a "lag") before air from the rock can reach the borehole, until which time pressure continues to decline. Eventually, pressure inside the borehole equilibrates with the fractured rock (i.e., rate of air flow from the rock formation is approximately equal to the rate of pumping), and pressure does not decline any more even if pumping continues.

During the lag period (when pressure is declining in response to pumping), the decrease in borehole temperature can be attributed to adiabatic expansion effects (see Equation 1). During this period, enthalpy of the air inside the borehole becomes smaller than that in 
the fractured formation. It is easy to see that borehole temperature (and enthalpy) will be at its minimum when borehole pressure just about reaches equilibrium with the pressure in the fracture formation (i.e., rate of flow of air into the borehole is just about equal to pumping rate). Once pressure has equilibrated, any mass of air that leaves the borehole because of pumping is replaced by an equivalent mass of air from the fracture formation. However, recall that the enthalpy of air leaving the borehole during this time is smaller than the enthalpy of air flowing into it. Consequently, once pressure reaches equilibrium, borehole temperature begins to increase. This process (of rising temperature) continues until equilibrium in enthalpy between the borehole and the fracture formation is established, after which time temperature does not climb any further even if pumping is continued (i.e., both pressure and temperature reaches steady state conditions). In other words, a turnaround (from falling to rising) in borehole temperature is observed during pumping in FFTL in a fracture formation. It is our hypothesis that this temperature signature can be utilized to estimate the transport properties of the fracture formation.

The temperature signature (the increase in temperature) after pumping stops can be explained similarly. At the end of pumping, air continues to flow in from the rock formation (because of residual pressure gradient) causing adiabatic compression (instead of previously observed adiabatic expansion) of air residing inside the borehole, which results in an instantaneous increase in borehole temperature. This process continues until (enthalpy) equilibrium is again established between the borehole and the fracture formation. In the following we demonstrate how the pressure and temperature data from FFTL can be used to determine the hydrologic properties of the surrounding fractured rock. We will also illustrate how FFTL data can be utilized to detect the location of highpermeable discrete fractures. However, before presenting any interpretation of actual FFTL data, we introduce a few concepts that will be helpful in understanding the analyses that follow.

A fractured rock formation normally consist of a rock matrix continuum (having low porosity and low permeability, a fracture continuum (consisting of many small and large interconnected fractures, with permeability which is considerably larger than that of the 
rock matrix continuum), and few discrete fractures with very large permeabilities (permeabilities that are larger than even that of the fracture continuum). To begin with, let us assume that the rock is not at all fractured (i.e. it has no fracture continuum or any discrete fracture), and that it is absolutely impervious (i.e., it has zero permeability). If air is pumped from a borehole located in such a rock, temperature (and pressure) in the borehole will decline continuously (because no air is supplied to the borehole from the rock which has zero permeability) until all the air is pumped out. Theoretically speaking (see Equation 1), as the last molecules are pumped out of the borehole, borehole temperature will approach absolute zero.

Let us now introduce a single high-permeable discrete fracture, which is narrow and thin, into the otherwise impervious rock. Because the discrete fracture has a very high permeability, it will bring in some amount of air into the borehole. However, this single discrete fracture, because it is thin and narrow, can bring in only a very small amount of air. Thus, the pressure and temperature in the borehole will decline in almost the same manner as in the previous case (no discrete fracture and impervious rock). If we plot temperature versus location in the borehole at different times, we will see rapidly declining temperatures at all locations except at the location of the discrete fracture, which will be held at more or less the constant initial temperature (because of its very fast communication with the boundary, resulting from its high permeability). As a result, the temperature data from FFTL can indicate the locations of high-permeability discrete fractures (for actual example of this, see discussion of Figure 6b).

The presence of the fracture continuum makes interpretation of FFTL data even more challenging. Because the fracture continuum has permeabilities larger than the matrix continuum, it can supply air into the borehole relatively quickly. At the same time, since the fractures in the fracture continuum are more numerous in number than the occasional large discrete fractures, they can supply substantially more mass of air into the borehole, compared to the few discrete fractures. As a result, FFTL performed in fractured rock produces the types of temperature signatures (initially declining and then increasing) that we discussed earlier in this section (and presented in Figure 2). Thus, transport properties 
estimated from FFTL data in fractured rock possibly provides an effective property (at the scale of measurement) of the fracture continuum, which may also include a few large discrete fractures.

Thus, FFTL can be useful in two ways. It can be used for detecting the presence of discrete fractures. FFTL can also be useful in estimation of permeability of the fracture continuum (i.e., if the rock is fractured with many small fractures in addition to some discrete high-permeable features). This is the main objective of this paper, i.e., to develop the theory for estimation of fracture continuum permeability from FFTL data. However, we will also illustrate how FFTL can be used to detect large discrete fractures.

\section{Single-Phase Conceptual Model}

The FFTL described in Section 2 was carried out in a borehole situated in unsaturated fractured rock. As such, an analysis of the temperature data will involve an analysis of unsaturated zone multiphase flow of air and water, as well as convective and conductive heat transfer. We defer the discussion of multiphase flow and heat transfer to Paper II. In this paper, we develop a simple conceptual model assuming that, in response to borehole pumping, dry air from the surrounding fractured rock flows into the borehole, i.e., we neglect the water and vapor phase present in the surrounding rock. In other words, it is assumed that single-phase, air-only conditions prevail in the entire system. In addition, we assume that the fractured rock can be represented as an effective continuum with a fixed porosity, and that air behaves like an ideal gas. We also assume that a Darcy flow regime is maintained throughout the FFTL. We will test the validity of this last assumption in Section 6.

The conceptual model for FFTL is schematically shown in Figure 3. The borehole has a radius of $R_{B}$ and a permeability of $k_{B}$, and a packed-off zone of length $H$ as shown in Figure 3. The borehole is surrounded by fractured rock with an effective continuum permeability of $k_{C}$. For the sake of simplicity and the semi-analytical solution that follows, we will assume that the borehole is intersected by one discrete fracture having a permeability of $k_{F}$, with the implicit assumption that $k_{B}>>k_{F}>>k_{C}$. The origin of the 
coordinate system is located at the bottom of the packed-off zone $(z=0)$. The discrete fracture is located at $z=h$, and pumping is applied at $z=H$, i.e., $H>h$. Constant pumping at the rate of $\dot{m}$ is applied at the top (i.e., $z=H$ ) of the packed-off zone. Before pumping starts (i.e., at $\mathrm{t}=0$ ), the fracture continuum, the discrete fracture, and the borehole are at constant temperature $T_{i}$ and constant pressure $P_{i}$. For this simplified conceptual representation, we also assume constant temperature and pressure conditions in the fractured rock and the discrete fracture throughout the duration of FFTL. This is reasonable given the large volume of the fracture continuum compared to the volume of air in the packed-off zone.

Let us consider a small time interval $\Delta t$ between $t=0$ (i.e., start of pumping) and $t=t+\Delta t$. When air is pumped from the packed-off zone over this small period of time $\Delta t$, a finite mass of air ( $=\dot{m} \Delta t$ ) leaves the packed-off zone in the borehole. Since a finite amount of air has left the packed-off zone, its pressure decreases (as does its temperature), creating a gradient between the borehole and the fracture continuum. Air that resides in the fracture continuum or in the discrete fracture flows into the packed-off zone driven by this gradient. However, since the permeability of the fractured rock is considerably smaller than that of the borehole, the rate at which air leaves the borehole initially is larger than the rate at which air can flow in from the rock formation. As a result, there is a net loss of air mass in the borehole initially. With passage of time, the difference between the rate of air mass leaving the borehole and flowing into it decreases, and pressure reaches an approximate steady state.

For this simplified system, assuming one-dimensional radial flow, the transient pressure behavior inside the borehole can be expressed as (see Appendix A for derivation)

$$
\frac{1}{\gamma \bar{P}_{B}} \frac{\partial P_{B}^{2}}{\partial t}=\frac{k_{B}}{\mu} \frac{\partial^{2} P_{B}^{2}}{\partial z^{2}}+\frac{2}{R_{B}^{2}} \frac{k_{C}}{\mu}\left(P_{C}^{2}-P_{B}^{2}\right)
$$

In Equation 2, $\gamma$ is the adiabatic coefficient for air (ratio of specific heat capacity of air at constant pressure and at constant volume), $P_{B}$ and $P_{C}$ are the pressures in the borehole and in the fracture continuum, and $\mu$ is the viscosity of air, which is assumed constant (given the typically small changes in pressure and temperature in FFTL, this is a 
reasonable assumption). Transient temperature response in the borehole can be described by (see Appendix A for derivation).

$$
-\frac{(\gamma-1)}{\gamma \bar{P}_{B}} \frac{\partial P_{B}^{2}}{\partial t}+2 P_{B} \frac{\partial \sigma_{B}}{\partial t}=-\frac{2}{R_{B}^{2}} \frac{k_{C}}{\mu}\left(P_{C}^{2}-P_{B}^{2}\right)\left(\frac{\sigma_{B}}{2}\right)+\frac{k_{B}}{\mu}\left(\frac{\partial P_{B}^{2}}{\partial z}\right)\left(\frac{\partial \sigma_{B}}{\partial z}\right)
$$

In Equation 3, $\sigma_{B}=\ln \frac{T_{B}}{T_{C}}$, where $T_{B}(z, t)$ is the temperature inside the borehole and $T_{C}$ is the temperature in the fracture continuum (assumed constant). Equations 2 and 3 are two equations with two variables, which need to be solved simultaneously for $P_{B}$ and $\sigma_{B}$ to determine the transient patterns of borehole pressure and temperature.

\section{Semi-Analytical Solution}

Before solving Equations 2 and 3, it is convenient to cast them into dimensionless form. The definition of the dimensionless variables and the actual solution steps along with the applicable boundary conditions, are provided in Appendix B. From Equation 2, the dimensionless pressure $\left(\psi_{B}\right)$ as a function of dimensionless radial location $(\zeta)$ and dimensionless time $(\tau)$ inside the borehole can be written as (see Appendix B for derivation)

$$
\psi_{B}(\zeta, \tau)=\dot{m}_{D}\left[\frac{1}{\varepsilon_{C B}}\left(1-e^{-\varepsilon_{C B} \tau}\right)+2 \sum_{n=1}^{\infty} \frac{(-1)^{n}}{n^{2} \pi^{2}+\varepsilon_{C B}}\left\{1-e^{\left(n^{2} \pi^{2}+\varepsilon_{C B}\right) \tau}\right\} \cos n \pi \zeta\right]
$$

In Equation 4, while the other symbols have their usual meanings, $\dot{m}_{D}$ is the dimensionless pumping rate given by

$$
\dot{m}_{D}=\frac{\dot{m}}{\pi R_{B}^{2}\left(\frac{k_{B} P_{C}}{\mu H}\right)\left(\frac{P_{C} M_{a}}{2 R T_{C}}\right)}
$$

and $\varepsilon_{C B}$ is given by

$$
\varepsilon_{C B}=\frac{2 H^{2}}{R_{B}^{2}}\left(\frac{k_{C}}{k_{B}}\right)
$$


Having obtained the analytical expression for $\psi_{B}$, we can now solve Equation 3 to obtain $\sigma_{B}$, the dimensionless borehole temperature. However, before solving Equation 3, it is helpful to introduce a new variable $y_{B}$ such that

$$
y_{B}=\ln \left(1-\psi_{B}\right)
$$

In terms of this new variable $y_{B}$, Equation 3 can be written as (see Appendix B)

$$
-(\gamma-1) \frac{\partial y_{B}}{\partial \tau}+2 \gamma \frac{\partial \sigma_{B}}{\partial \tau}=\varepsilon_{C B}\left(\frac{\sigma_{B}}{2}\right)\left(1-e^{-y_{B}}\right)+\left(\frac{\partial y_{B}}{\partial \zeta}\right)\left(\frac{\partial \sigma_{B}}{\partial \zeta}\right)
$$

Before proceeding further, note a special feature of Equation 8. If $\varepsilon_{C B}=0$ (i.e., if there is no communication between the borehole and the fractured rock) and if pumping is performed so slowly such that equilibrium is maintained at all times (i.e., $\frac{\partial y_{B}}{\partial \zeta} \approx 0 ; \frac{\partial \sigma_{B}}{\partial \zeta} \approx 0$ ), Equation 8 reduces to

$$
\frac{\partial \sigma_{B}}{\partial \tau}=\frac{\gamma-1}{2 \gamma} \frac{\partial y_{B}}{\partial \tau}
$$

which can be further simplified to (subject to the initial condition given in Equation B.9a)

$$
\sigma_{B}=\frac{\gamma-1}{2 \gamma} y_{B}
$$

After some algebraic manipulation, one can write Equation 10 as

$$
\frac{T_{B}}{T_{C}}=\left(\frac{P_{B}}{P_{C}}\right)^{\frac{\gamma-1}{\gamma}}
$$

Equation 11 is the adiabatic pressure-temperature relationship (see Equation 1) for a reversible process involving ideal gases. In other words, as expected, Equation 8 reproduces the adiabatic, reversible pressure-temperature relationship for ideal gases under truly constant volume conditions.

Since $\psi_{B}$ (and hence $y_{B}$ ) is explicitly known from Equation 4, we can now obtain a solution for Equation 8. While Equation 2 could be solved explicitly for $\psi_{B}$, no explicit 
analytical solution can be developed for Equation 8, because of the presence of the nonlinear terms (particularly because of the presence of the product of the two derivative terms, which cannot be ignored). We thus propose a fully implicit finite difference solution for Equation 8. In other words, we can write

$$
\sigma_{B, i}^{n+1}=\frac{\frac{\gamma-1}{\Delta \tau}\left[y_{B, i}^{n+1}-y_{B, i}^{n}\right]+\frac{2 \gamma}{\Delta \tau} \sigma_{B, i}^{n}-\frac{1}{\Delta \zeta}\left(\frac{\partial y_{B}}{\partial \zeta}\right)_{i}^{n+1} \sigma_{B, i-1}^{n}}{\frac{2 \gamma}{\Delta \tau}-\frac{1}{\Delta \zeta}\left(\frac{\partial y_{B}}{\partial \zeta}\right)_{i}^{n+1}-\frac{\varepsilon_{C B}}{2}\left[1-\exp \left(-y_{B, i}^{n+1}\right)\right]}
$$

In Equation 12, subscripts “ $i$ ” and “ $i-1$ ” refer to spatial location; superscript " $n$ ” refer to conditions in previous time step and superscript " $n+1$ ” to conditions in the next time step. Equation 12 can be solved numerically, subject to the boundary condition given in Equation B.9b (see Appendix B). This boundary condition implies that the permeability of the discrete fracture is (relatively) large compared to the rest of the fractured rock, so that it can communicate with the borehole almost instantaneously. In other words, the temperature inside the borehole at the location of the discrete fracture always remains invariant (for this simplified conceptual representation).

\section{Analysis of Synthetic FFTL Response}

To facilitate verification of the semi-analytical solution and the subsequently estimated parameters, a synthetic FFTL experiment with known input parameters was simulated using the TOUGH2 simulator [Pruess, 1992; Pruess et al., 1999], which is a generalpurpose software for simulating multiphase, multicomponent flow and heat transport in porous and fractured media. Figure 3a shows the pressure and temperature response from this synthetic FFTL experiment. The pumping rate for this synthetic FFTL, and the input parameters, are listed in Table 1. In this synthetic experiment, air was pumped from a $0.08 \mathrm{~m}$ diameter borehole for a period of 326 seconds at the rate of approximately $0.55 \times 10^{-3} \mathrm{~kg} / \mathrm{s}$ ( $\left.\sim 30 \mathrm{SLPM}\right)$. Other input parameters needed for the analytical solution are also given in Table 1. The analytical solutions developed using Equations 4 (for pressure) and 12 (for temperature) are compared with TOUGH2 simulator predictions. 
Before proceeding further, we would like to test the validity of the Darcy (or laminar) flow regime for this synthetic FFTL. To do this, we need an estimate of the Reynolds number for flow inside the borehole. The velocity of air $(V)$ inside the borehole resulting from pumping of air at the rate of $\dot{m}$ can be approximated from (the actual velocity may be slightly larger because of the reduction of flow area owing to the space occupied by the logging tool)

$$
V=\frac{\dot{m}}{\pi R_{B}^{2} \rho}
$$

The Reynolds number then can be estimated from

$$
\operatorname{Re}=\frac{2 R_{B} V \rho}{\mu}
$$

Using Equation 13 in Equation 14 to eliminate V, we obtain

$$
\mathrm{Re}=\frac{2 \dot{m}}{\pi R_{B} \mu}
$$

For the synthetic FFTL, $\dot{m}=0.55 \times 10^{-3} \mathrm{~kg} \mathrm{~s}^{-1}, R_{B}=0.038 \mathrm{~m}$, and $\mu=1.86 \times 10^{-5} \mathrm{~kg} \mathrm{~m}^{-1}$ $\mathrm{s}^{-1}$ (see Table 1), we thus obtain a Reynolds number of approximately 250. The actual Reynolds number may be slightly larger than this because of the reduction of flow space owing to the logging tool volume. However, laminar flow is expected to occur in a pipe as long as the Reynolds number is less than 2100-2300. It is unlikely that the Reynolds number will be close to 2100-2300, even if corrections are made for the reduction in flow space. Further, the actual pumping rate used for the FFTL data presented in Figure 2 was smaller than $0.55 \times 10^{-3} \mathrm{~kg} \mathrm{~s}^{-1}$, which will lead to a Reynolds number smaller than 250 . Since, even for the largest rate of pumping, the estimated Reynolds number is only about 250, it can be safely assumed that laminar flow conditions (and a Darcy flow regime) is maintained during the FFTL presented in this paper.

Since our first objective is to analyze the physical processes that occur immediately after commencement of pumping, Figure 4a shows the pressure and temperature response for relatively small times (less than 5 seconds). Comparing the analytical solutions to TOUGH2 results during this early time period after pumping began, it can be said that the analytical solution is reasonably accurate. For example, the maximum difference between 
the semi-analytical solution and TOUGH2 predictions happens at a time when temperature is at its minimum. At that time, the semi-analytical solution (see Figure 4a) predicts a temperature drop of $\sim 0.57^{\circ} \mathrm{C}$ (starting from an initial temperature of $24^{\circ} \mathrm{C}$ ), whereas the TOUGH2 simulations predict a temperature drop of $0.6^{\circ} \mathrm{C}$ (from the same initial temperature). The absolute value of maximum relative error is thus about $5 \%$ $[100 \times(0.6-0.57) / 0.6]$. At any other time, the absolute relative error is smaller $(\sim 1-2 \%)$.

Pressure declines rapidly over a period of about 0.5 second before asymptotically reaching a steady-state situation. The initial decline in pressure is because more air is pumped out than is coming in from the formation. Once a steady state is reached (with the rate of pumping approximately equal to the overall rate of flow coming into the borehole from outside), pressure changes only marginally. This explains the initial physics of flow between the borehole and the fracture continuum.

Pressure and temperature response from the same synthetic FFTL over a longer time period is shown in Figure 4b. The figure compares analytical and TOUGH2 simulation results over the first 30 seconds after pumping started. Several inferences can be drawn from Figure 4b. First, analytical solutions compare well with TOUGH2 predictions over the entire time period in which thermal equilibrium is attained between the borehole and the fracture continuum outside (the difference between semi-analytical solution and TOUGH2 prediction is smaller at larger times). This increases confidence in the analytical solution. Second, from both the analytical solutions and TOUGH2 simulations, it is obvious that temperature declines until the pressure declines with pumping. However, once the pressure reaches a steady state (after sufficient fluid communication has been established between the borehole and the fracture continuum), temperature starts to increase. The time needed for temperature to equilibrate is controlled by the rate of pumping, and the difference in enthalpy of air inside the borehole and in the fracture formation. How fast enthalpy equilibration between the two will be accomplished is controlled by the parameter $\varepsilon_{C B}$ (see Equation 6), which represents the ratio of fracture continuum and borehole permeabilities multiplied by the square of the characteristic length scale of the transport problem (i.e., ratio of the length of the packed-off zone and 
the radius of the borehole). In other words, $\varepsilon_{C B}$ represents the ease of communication between the borehole and the fracture continuum.

To illustrate the influence of $\varepsilon_{C B}$ on FFTL response, we repeated the synthetic FFTL for three different values of the dimensionless parameter $\varepsilon_{C B}\left(\varepsilon_{C B}=0.005,0.0005\right.$, and 0.000125). Pressure and temperature response from these three synthetic FFTLs are shown in Figure 5. Note that the results in Figure 5 are obtained using Equations 4 and 12 , and not through simulations using TOUGH2. With decreasing values of the parameter $\varepsilon_{C B}$, the decline in pressure and temperature is steeper. Also, for smaller values of the parameter $\varepsilon_{C B}$, it takes temperature longer to return to its pre-pumping values. These observations make sense because a smaller value of the parameter implies a smaller permeability for the fracture continuum (see Equation 6) for a given FFTL setup. A smaller (or larger) permeability in turn implies that air can move in relatively slowly (or quickly) in response to pumping, resulting in sharper (or smaller) initial temperature drops. Since the temperature response can be directly correlated to transport properties (particularly permeability) of the outside continuum, temperature logging data can be utilized to get an estimate of the transport properties of the host rock.

In addition, we can also use the temperature response from a logging experiment to locate a high-permeability discrete feature. Consider Figure 6a as an example. In Figure 6a, temperature profiles along the borehole are shown from a synthetic FFTL at different times. In this synthetic FFTL, the parameter $\varepsilon_{C B}$ is set as 0.005. A discrete feature is present in this experiment at a location of $z=1.0 \mathrm{~m}$ (the bottom of the borehole is at $z=$ $0.0 \mathrm{~m}$ and the top is at $z=2.0 \mathrm{~m}$ ). From Figure 6a, observe that a spike in temperature exists (without any cooling) at small times ( $<1.0$ second) at the location of the discrete fracture, whereas temperature is mostly uniform elsewhere in the borehole (with general cooling inside the borehole at these early times because of pumping). With continued pumping, temperature at the location of the discrete fracture begins to equilibrate with the rest of the borehole, resulting in a slow disappearance of the spike. How long such a spike in temperature (owing to the presence of a discrete feature) exists depends on the 
properties of the formation, as illustrated in Figure 6b, which shows the temperature profiles in the borehole at 10.2 seconds after pumping started for three different values of the parameter $\varepsilon_{C B}$. Notice that the smaller the value of the parameter $\varepsilon_{C B}$, the longer the spike, owing to a discrete feature existing in the temperature response from a logging experiment.

The observations from these synthetic FFTLs can be summarized as follows. The initial drop (and subsequent rise) in temperature can be correlated to the transport properties of the host rock. The smaller the permeability of the host rock, the larger the initial drop in temperature and the longer it will take to eventually return to its initial conditions. If the permeability of the host rock is sufficiently low $\left(\varepsilon_{C B} \sim 0\right)$, temperatures will return to prepumping conditions after a very long time. In addition, the presence of a discrete feature can be detected by looking at the vertical temperature profile inside the borehole soon after pumping has begun.

\section{Estimation of Permeability}

To determine the transport properties of the host rock from the temperature logging data, we propose an approximate method, which provides a first-order estimate of the parameter $\varepsilon_{C B}$. Since the permeability of the fracture continuum can be estimated once $\varepsilon_{C B}$ is known (see Equation 6), this proposed method provides a first-order estimate of the continuum permeability from the temperature logging data. This first-order estimate then can be further refined through actual inversion of the temperature and pressure data, using numerical optimization software such as iTOUGH2 [Finsterle, 2004]. The inversion of actual FFTL data is described in Paper II [Mukhopadhyay et al., 2008].

For the simple conceptual model presented in Section 4 , the rate $\left(\dot{m}_{i n}\right)$ at which air flows into the borehole from the fracture continuum at any dimensionless time $\tau$ can be written as (see Appendix C for derivation)

$$
\dot{m}_{\text {in }}=\dot{m}\left[1-\exp \left(-\varepsilon_{C B} \tau\right)\right]
$$


where, as noted previously, $\dot{m}$ (in $\mathrm{kg} / \mathrm{s}$ ) is the rate of pumping at the borehole (assumed constant). Thus, at any given time $\tau$, only a fraction of the mass of air that is pumped out gets refilled by flow of air from the fractured rock. Only as $\tau \rightarrow \infty$ is a true steady state reached, after which pressure does not decline any further with time and temperature begins to climb back towards its pre-pumping condition. However, for all practical purposes, it can be assumed that a steady state has been obtained when $\dot{m}_{\text {in }} / \dot{m} \approx 0.99$. With such an approximation, one can write (using Equation 15)

$$
\varepsilon_{C B}=-\frac{\ln (0.01)}{\tau_{c}}
$$

In Equation 16, $\tau_{c}$ is defined as the critical dimensionless time at which steady state is approximately reached, such that temperature begins its upward climb (or pressure reaches its steady-state condition).

Using the definitions of the dimensionless variables, Equation 16 can be written in terms of real variables as follows

$$
k_{C} \approx 2.303 \frac{\mu R_{B}^{2}}{\gamma \bar{P}_{B} t_{c}}
$$

Since the viscosity of air $(\mu)$, the borehole diameter $\left(R_{B}\right)$, the ratio of specific heat capacities of air $(\gamma)$, and the average borehole pressure $\left(\overline{P_{B}}\right)$ are known, $k_{C}$ can be determined if $t_{c}$ is known. From the temperature signature of FFTL, $t_{c}$ can be obtained as the time at which there is a turnaround (i.e., the time at which $\partial T_{B} / \partial t$ changes from negative to positive) in a temperature-versus-time plot.

The proposed estimation method is applied to the synthetic FFTL data presented in Figure 4a. Figure 7 shows the temperature signature from the same synthetic FFTL, as in Figure 4a. Figure 7 also shows the ratio of inflow rate of air into the borehole and the pumping rate (i.e., $\dot{m}_{i n} / \dot{m}$ ) as a function of time for this experiment (estimated using Equation C.7). From Figure 7, we note that $t_{c}$ is 0.49 seconds for this experiment. Using 
the values $\mu=1.86 \times 10^{-5} \mathrm{~kg} \mathrm{~m}^{-1} \mathrm{~s}^{-1}, R_{B}=0.04 \mathrm{~m}, \gamma=1.4$, and $\bar{P}_{B}=99650 \mathrm{~Pa}$ (approximately), we obtain (using Equation 17) $k_{C}=1.0 \times 10^{-12} \mathrm{~m}^{2}$, which is the input permeability of the fracture continuum for this synthetic FFTL (see Table 1). In other words, Equation 17 can be used to compute the permeability of the fracture continuum. This finding illustrates that temperature data from a logging experiment can be used for determining fracture continuum permeability of the host medium.

The fracture continuum permeability for the synthetic FFTL was known. In an actual FFTL, the fracture continuum permeability is unknown and is one of the transport parameters we want to estimate. To illustrate how this can be accomplished, we return to the actual FFTL data presented in Figure 2b. For this FFTL, observe that temperature decreases for about six seconds after pumping began, i.e., $t_{c}=6.0$ seconds. From Figure 2a, it can also be estimated that the average pressure $\bar{P}_{B}=75800$ Pa. For this particular FFTL, $R_{B}=0.038 \mathrm{~m}$ (the diameter of the borehole is $0.076 \mathrm{~m}$ ). With typical values of $\mu$ $=1.86 \times 10^{-5} \mathrm{~kg} \mathrm{~m}^{-1} \mathrm{~s}^{-1}$ and $\gamma=1.4$, the effective fracture continuum permeability is estimated to be $3.9 \times 10^{-13} \mathrm{~m}^{2}$ (using Equation 17). From previous air-injection tests, the fracture continuum permeability of the DST host rock has been found to vary almost three orders of magnitude between $1.6 \times 10^{-15}$ and $9.7 \times 10^{-13} \mathrm{~m}^{2}$, with a geometric mean of $1.3 \times 10^{-13} \mathrm{~m}^{2}$ [Birkholzer and Tsang, 2000; Freifeld, 2001]. Thus, the estimate of fracture continuum permeability from Equation 17 compares well with the mean of the previous estimates of fracture continuum permeability for the DST host rock. Equation 17 thus provides a useful tool with which to obtain a reasonable estimate of the fracture continuum permeability from FFTL response in unsaturated fractured rock. The estimate could possibly be further refined, when a more comprehensive model of multiphase transport is utilized. We will elaborate on this in Paper II [Mukhopadhyay et al., 2008]. In Paper II [Mukhopadhyay et al., 2008], we will also show that FFTL data can be used to estimate transport parameters other than permeability as well.

\section{Summary}


Flowing fluid temperature logging, or FFTL is a relatively new concept that, when backed up by the emerging technology of distributed temperature sensing, or DTS, has strong potential as a viable tool for locating flowing fractures and estimating the transport parameters associated with fracture flow. We have developed the necessary theoretical background for interpretation of FFTL data from unsaturated fractured rock. In this paper, we present a simple conceptual model of FFTL based on the dual assumptions of single-phase flow of air (i.e., ignoring the flow of water vapor) and constant pressure and temperature inside the rock formation (i.e., assuming that the perturbation in the rock formation caused by short-duration FFTL is negligible). It is also assumed that heat transfer via convection only, i.e., conductive heat transfer between the borehole and the rock formation is ignored. Based on this simplified conceptualization of FFTL, we develop a semi-analytical solution for spatial and temporal variation in pressure and temperature inside the borehole, in response to an applied perturbation in the form of pumping of air.

The semi-analytical solution is compared with predictions from the TOUGH2 numerical simulator. It was observed that the semi-analytical solution compares reasonably well with the numerical results. We used the semi-analytical solution to investigate the parameter (i.e., permeability) that has the strongest influence on pressure and temperature response from FFTL. We also illustrate how the FFTL temperature response can be utilized to detect the location of discrete features embedded in what is otherwise a fracture continuum.

Based on the semi-analytical solution, we propose a method for estimating the effective fracture continuum permeability. Our proposed method accurately reproduced the fracture continuum permeability from temperature data of a synthetic FFTL experiment with known permeability. We subsequently applied the proposed method to estimation of fracture continuum permeability from temperature data of an actual FFTL conducted in the host rock of the Drift-Scale Test at Yucca Mountain, Nevada. The effective fracture continuum permeability estimated from the proposed method is comparable to previous independent estimates of fracture permeability. 
The borehole pressure and temperature response from FFTL in unsaturated fractured rock communicate the underlying multiphase flow and heat transfer processes in response to the applied perturbation (i.e., pumping of air from the borehole). While we present a simplified conceptualization of FFTL in this paper, a more elaborate numerical model of FFTL is presented in Paper II, where we perform a systematic sensitivity study to determine the parameters (such as permeability, porosity, and thermal conductivity) having the strongest influence on FFTL response, and estimate these parameters using multiphase inverse modeling based on the iTOUGH2 optimization code.

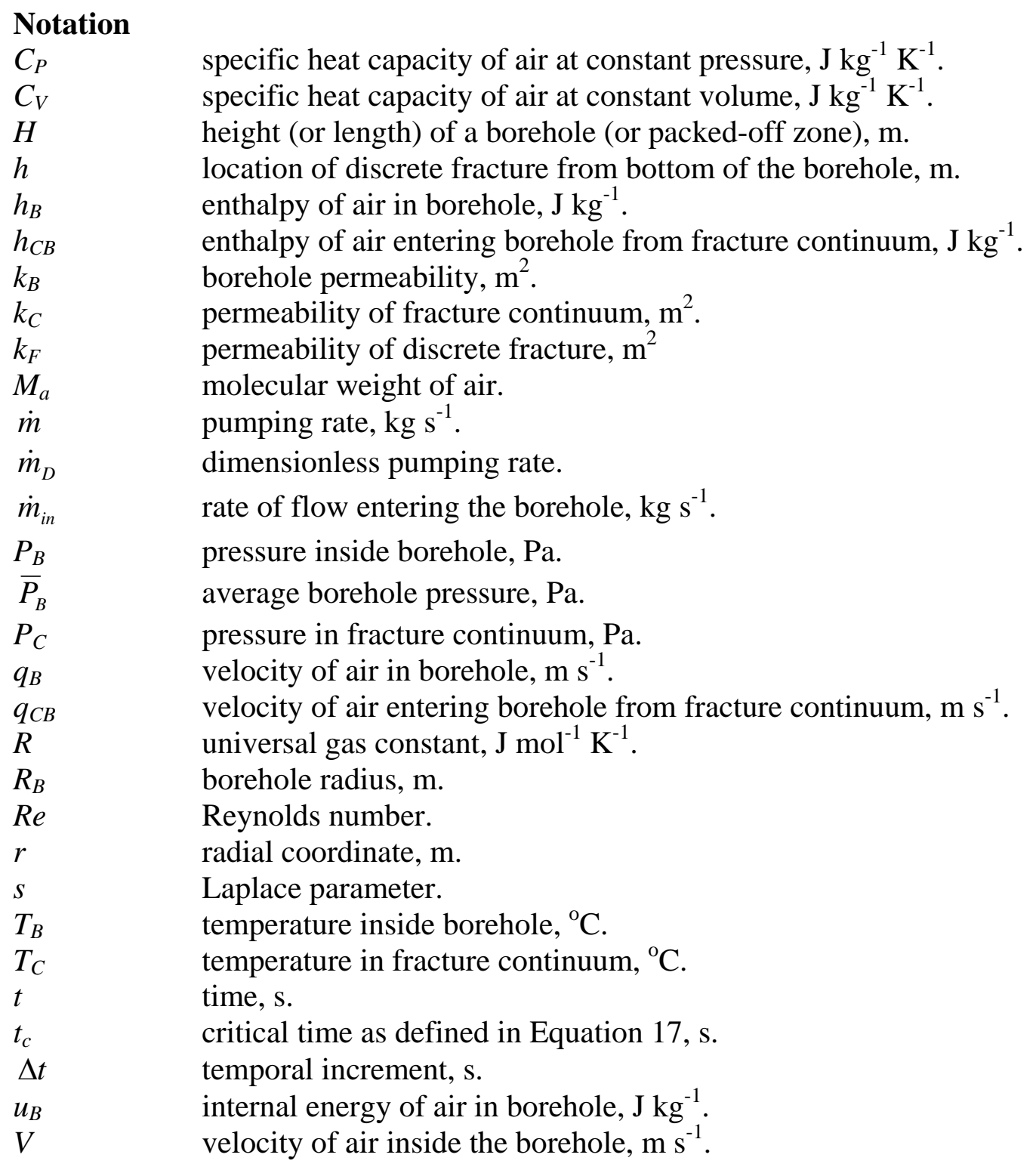




$\begin{array}{ll}y_{B} & \text { logarithm of dimensionless pressure in the borehole. } \\ Z & \text { z-coordinate, } \mathrm{m} . \\ \Delta z & \text { spatial increment in the z-direction, } \mathrm{m} . \\ \text { Greek Symbols } & \text { momentum diffusivity for air in borehole, } \mathrm{m}^{2} \mathrm{~s}^{-1} \text {. } \\ \alpha_{B} & \text { dimensionless interaction parameter between borehole and fracture } \\ \varepsilon_{C B} & \text { continuum, see Equation } 6 . \\ \gamma & \text { ratio of } C_{P} \text { and } C_{V} \text { for an ideal gas } \\ \mu & \text { viscosity of air, kg } \mathrm{m}^{-1} \mathrm{~s}^{-1} \text {. } \\ \rho_{B} & \text { density of air in borehole, kg } \mathrm{m}^{-3} \text {. } \\ \rho_{C B} & \text { density of air entering borehole from fracture continuum, } \mathrm{kg} \mathrm{m}^{-3} . \\ \sigma_{B} & \text { logarithm of dimensionless pressure. } \\ \tau & \text { dimensionless time. } \\ \tau_{c} & \text { dimensionless critical time as defined by Equation } 16 . \\ \psi_{B} & \text { dimensionless borehole pressure. } \\ \bar{\psi}_{B} & \text { dimensionless borehole pressure in Laplace transform space. } \\ \psi_{C} & \text { dimensionless fracture continuum pressure. } \\ \zeta & \text { dimensionless distance in the } \mathrm{z} \text {-direction. } \\ \zeta_{F} & \text { location of discrete fracture in dimensionless space. }\end{array}$

\section{Acknowledgment}

We thank the anonymous reviewers for their careful and critical review of the manuscript. We thank Stefan Finsterle and Dan Hawkes of the Ernest Orlando Lawrence Berkeley National Laboratory (Berkeley Lab) for their constructive review of the draft manuscript. We also thank Chin-Fu Tsang and Paul Cook of the Berkeley lab for sharing the photographs of the FFTL instrument (Figure 1) and the FFTL data (Figure 2) with us. Diana M. Swantek of the Berkeley Lab prepared the graphics for Figure 3, and her contribution is duly acknowledged. This article has been authored by Berkeley Lab under Contract No. DE-AC02-05CH11231 with the U.S. Department of Energy. The United States Government retains and the publisher, by accepting the article for publication, acknowledges that the United States Government retains a non-exclusive, paid-up, irrevocable, world-wide license to publish or reproduce the published form of this manuscript, or allow others to do so, for United States Government purposes. The views expressed in this article are those of the authors and do not necessarily state or reflect the views or policies of the United States Government or any agency thereof or The Regents of the University of California. 


\section{References}

Abramowitz, M., and I.A. Stegun (eds.) (1964), Handbook of Mathematical Functions, National Bureau of Standards, Washington, District of Columbia.

Benoit, D., and S. Thompson (1998), Development and testing of a single-ended distributed temperature sensing system at the Beowawe, Nevada geothermal reservoir, GRC Trans., 22, 575-581.

Birkholzer, J.T., and Y.W. Tsang (2000), Modeling the thermal-hydrologic processes in a large-scale underground heater test in partially saturated fractured tuff, Water Resour. Res., 36(6), 1431-1447.

Blackwell, D.D., G.R. Beardsmore, R.K. Nishimori, and M.J. McMullen, Jr. (1999), High-resolution temperature logs in a petroleum setting, examples and applications, in A. Förster and D. Merriam (eds.), Geothermics in basin analysis, Kluwer Academic/Plenum Publishers, New York, NY, 1-33.

Chisholm, T.J., and D.S. Chapman (1992), Climate change inferred from analysis of borehole temperature: An example from western Utah, Journal of Geophysical Research, 97, 14,155-14,175.

Constanz, J., S.W. Tyler, and E. Kwicklis (2003), Temperature-profile methods for estimating percolation rates in arid environments, Vadose Zone Journal, 2, 12-24.

Doughty, C. and C.-F. Tsang (2005), Signatures in flowing fluid electric conductivity logs, Journal of Hydrology, 310, No. 1-4, 157-180.

Doughty, C., S. Takeuchi, K. Amano, M. Shimo, and C.-F. Tsang (2005), Application of multi-rate flowing fluid electric conductivity logging method to Well DH-2, Tono Site, Japan, Water Resour. Res., 41, W1041, 10.1029/2004WR003708.

Drury, M.J., A.M. Jessop, and T.J. Lewis (1984), The detection of groundwater flow by precise temperature measurements in boreholes, Geothermics, 13, 163-174.

Finsterle, S. (2004), Multiphase inverse modeling: Review and iTOUGH2 applications, Vadose Zone J., 3, 747-762.

Freifeld, B.M. (2001), Estimation of fracture porosity of an unsaturated fractured welded tuff using gas tracer testing, Ph.D. Thesis, University of California, Berkeley, Calif.

Förster, A., J. Schrötter, D.F. Merriam, and D.D. Blackwell (1997), Application of optical-fiber temperature logging - an example in a sedimentary environment, Geophysics, 62(4), 1107-1113. 
Ge, S. (1998), Estimation of groundwater velocity in localized fracture zones from well temperature profiles, Journal of Volcanology and Geothermal Research, 84, 93-101.

Harris, R.N., and D.S. Chapman (1995), Infering surface ground temperature histories from borehole temperature measurements in the Colorado Plateau of eastern Utah, Journal of Geophysical Research, 100, 6367-6381.

Harris, R.N., and D.S. Chapman (2007), Stop-go temperature logging for precision applications, Geophysics, 62(4), E119-E123.

Henninges J., E. Huenges, H. Burkhardt (2005), In situ thermal conductivity of gashydrate-bearing sediments of the Mallik 5L-38 well, J. Geophys. Res., 110, B11206, doi:10.1029/2005JB003734.

Huang, K., Y.W. Tsang, and G.S. Bodvarsson (1999), Simultaneous inversion of airinjection tests in fractured unsaturated tuff at Yucca Mountain, Water Resour. Res., 35(8), 2375-2386.

Hurtig, E., J. Schrötter, S. Großwig, K. Kühn, B. Harjes, W. Wieferg, and R.P. Orrell (1993), Borehole temperature measurements using distributed fibre optic sensing, Scientific Drilling, 3 (6), 283-286.

Hurtig, E., S. Großwig, M. Jobmann, K. Kühn, and P. Marschall (1994), Fiber-optic temperature measurements in shallow boreholes: experimental applications for fluid logging, Geothermics, 23(4), 355-364.

Ikeda, N. (2003), Fracture reservoir characterization by fiber optic distributed temperature log, Proc. Twenty-Eighth Workshop on Geothermal Engineering, Stanford University, Stanford, Calif.

Ikeda, N., U. Kohji, S. Kawazoe, and K. Haruguchi (2000), Delineation of fracture reservoir by transient temperature analysis using fiber optic sensor, Proc. World Geothermal Congress 2000, 2617-2621, Kyushu-Tokuhu, Japan.

Jessop, A.M. (1990), Thermal geophysics, Elsevier Science Publication Company, Inc., New York, NY, 306 p.

Kirkham, D. (1946), Field method for determination of air permeability of soil in its undisturbed state, Soil. Sci. Soc. Am. Proc., 11, 93-99, 1946.

Mukhopadhyay, S., and M. Sahimi (2000), Calculation of the effective permeabilities of a field-scale heterogeneous porous medium, Chemical Engineering Science, 55, 44954513. 
Mukhopadhyay, S., and Y.W. Tsang (2003), Uncertainties in coupled thermalhydrological processes associated with the Drift-Scale Test at Yucca Mountain, Nevada, Journal of Contaminant Hydrology, 62-63, 595-612.

Mukhopadhyay, S., Y.W. Tsang, and J.T. Birkholzer (2007), Estimation of field-scale thermal conductivities of unsaturated fractured rocks from in situ temperature data, Water Resour. Res., 43, W09418, doi:10.1029/2006WR005283.

Mukhopadhyay, S., Y. W. Tsang, and S. Finsterle (2008), Parameter estimation from flowing fluid temperature logging data in unsaturated fractured rock using multiphase inverse modeling, Water Resour. Res. (submitted).

Osato, K., S. Takasugi., S. Osawa, K. Hashiba, and K. Perales (1995), Temperature profiling/bottom pressure monitoring system using optical fiber and capillary tube - field test in a geothermal well, Proc. World Geothermal Congress 1995, 1921-1926, Florence, Italy.

Prensky, S. (1992), Borehole temperature measurements: An overview of engineering and scientific applications, The Log Analyst, 33(3), 313-333.

Pruess, K. (1991), TOUGH2-A General-Purpose Numerical Simulator for Multiphase Fluid and Heat Flow, LBNL-29400, Lawrence Berkeley Natl. Lab., Berkeley, Calif.

Pruess, K., C.M. Oldenburg, and G.J. Moridis (1999), TOUGH2 User's Guide, Version 2.0, LBNL-43134, Lawrence Berkeley Natl. Lab., Berkeley, Calif.

Sakaguchi, K. and N. Matsushima (1995), Temperature profile monitoring in geothermal wells by distributed temperature sensing technique, GRC Trans., 19, 355-358.

Sakaguchi, K. and N. Matsushima (2000), Temperature logging by the distributed temperature sensing technique during injection tests, Proc. World Geothermal Congress 2000, 1657-1661, Kyusu-Tohuku, Japan.

Selker, J.S., L. Thévenaz, H. Huwald, A. Mallet, W. Luxemburg, N. van de Giesen, M. Stejskal, J. Zeman, M. Westhoff, and M.B. Parlange (2006), Distributed fiber-optic temperature sensing for hydrologic systems, Water Resour. Res., 42, W12202, doi: 10.1029/2006WR005326.

Smith, L., and D.S. Chapman (1983), On the thermal aspects of groundwater flow. I: Regional scale systems, Journal of Geophysical Research, 88, 593-608.

Smith, J.M., and H.C. Van Ness (1981), Introduction to Chemical Engineering Thermodynamics, $3^{\text {rd }}$ edition ( $9^{\text {th }}$ printing), McGraw-Hill International Book Company, Tokyo, Japan. 
Sonnenthal, E.L., C.F. Ahlers, and G.S. Bodvarsson (1997), Fracture and fault properties for the UZ site-scale flow model, in The Site-Scale Unsaturated Zone Model of Yucca Mountain, Nevada, for the Viability Assessment, LBNL-40376, Lawrence Berkeley Natl. Lab., Berkeley, Calif.

Tsang, C.-F., and C. Doughty (2003), Multirate flowing fluid electric conductivity logging method, Water Resour. Res., 39, 12, 1354-1362, doi:10.1029/2003WR002308.

Tsang, C.-F., P. Hufschmied, and F. V. Hale (1990), Determination of fracture inflow parameters with a borehole fluid conductivity logging method, Water Resour. Res., 26(4) 561-578, 1990.

Tsang, C.-F, F.V. Hale, P. Bluemling, and S. Vomvoris (1994), Borehole fluid dynamic temperature logging to evaluate fracture hydraulic properties, presented at International High-Level Radioactive Waste management Conference, May 22-26, 1994, Las Vegas, Nevada.

Tsang, C.-F., J. Clyde, P.J. Cook, and R. Solbau (2007), Flowing fluid temperature logging method for determining hydrologic properties of unsaturated fractured rocks, LBNL-63564, Lawrence Berkeley Natl. Lab., Berkeley, Calif.

Wisian, K. W., D.D. Blackwell, S. Bellani, J.A. Henfling, R.A. Normann, P.C. Lysne, A. Förster, and J. Schröter (1998), Field comparison of conventional and new technology temperature logging system, Geothermics, 27 (2), 131-141.

Yortsos, Y.C., and J. Chang (1990), Capillary effects in steady-state flow in heterogeneous cores, Transp. Porous Media, 5, 399-420.

\section{Appendix A}

Considering an elementary volume of $\pi R_{i}^{2} \Delta z$ of the borehole between $z$ and $z+\Delta z$, the equation of continuity can be written as (neglecting the small mass of air flowing in through the single, narrow discrete fracture)

$\left.\left.\pi R_{B}^{2} \Delta z \rho_{B}\right|^{t+\Delta t}-\left.\pi R_{B}^{2} \Delta z \rho_{B}\right|^{t}=\Delta t\left|\pi R_{B}^{2} q_{B} \rho_{B}\right|_{z}-\left.\pi R_{B}^{2} \Delta z q_{B} \rho_{B}\right|_{z+\Delta z}+2 \pi R_{B} \Delta z q_{C B} \rho_{C B}\right]$.

With both $\Delta z \rightarrow 0$ and $\Delta t \rightarrow 0$, Equation A1 can be written in differential notation as

$$
\frac{\partial \rho_{B}}{\partial t}=-\frac{\partial}{\partial z}\left(q_{B} \rho_{B}\right)+\frac{2}{R_{B}}\left(q_{C B} \rho_{C B}\right) .
$$


Assuming Darcy flow, we can write the flow rates $q_{B}$ (the rate of flow within the borehole) and $q_{C B}$ (the rate of flow into the borehole from the surrounding porous continuum) as

$$
q_{B}=-\frac{k_{B}}{\mu} \frac{\partial P_{B}}{\partial z}
$$

\section{(A.3a)}

and

$$
q_{C B}=\frac{k_{C}}{\mu}\left(\frac{P_{C}-P_{B}}{R_{B}}\right)
$$

In Equation A.3a, it is assumed that pressure changes linearly from $P_{C}$ to $P_{B}$ over a radius of influence, which is equal to the radius of the borehole (i.e., it is assumed that the constant pressure and temperature boundary is located at a distance of $R_{B}$ from the borehole wall). Assuming air behaves like an ideal gas, we have $\rho_{B}=\frac{P_{B} M_{a}}{R T_{B}}$ and $\rho_{C B}=\frac{\left(P_{C}+P_{B}\right) M_{a}}{\left(T_{C}+T_{B}\right) R}$, where $M_{a}$ is the molecular weight of air, and $R$ is the universal gas constant (8.314 J/mol-K). Introducing these definitions in Equation A.2, we obtain

$$
\frac{\partial}{\partial t}\left(\frac{P_{B}}{T_{B}}\right)=\frac{k_{B}}{\mu} \frac{\partial}{\partial z}\left(\frac{P_{B}}{T_{B}} \frac{\partial P_{B}}{\partial z}\right)+\frac{2}{R_{B}^{2}} \frac{k_{C}}{\mu}\left(\frac{P_{C}^{2}-P_{B}^{2}}{T_{C}+T_{B}}\right) .
$$

In writing Equation A.4, we have assumed that air viscosity does not change with pressure and temperature. Since the changes in temperature and pressure during a dynamic fluid logging experiment are expected to be small, this is a reasonable assumption.

Expanding the partial differentials in Equation A.4, after some rearrangement, we can rewrite Equation A.4 as

$$
\frac{\partial P_{B}}{\partial t}-P_{B} \frac{\partial \ln T_{B}}{\partial t}=\frac{k_{B}}{\mu}\left[\frac{1}{2} \frac{\partial^{2} P_{B}^{2}}{\partial z^{2}}-\frac{1}{2}\left(\frac{\partial P_{B}^{2}}{\partial z}\right)\left(\frac{\partial \ln T_{B}}{\partial z}\right)\right]+\frac{2}{R_{B}^{2}} \frac{k_{C}}{\mu}\left(\frac{T_{B}}{T_{C}+T_{B}}\right)\left(P_{C}^{2}-P_{B}^{2}\right)
$$

Assuming further that the properties can be evaluated at some average pressure $\bar{P}_{B}$ (say, the average of the initial pressure and the steady state pressure in the borehole), Equation 
A.5 can be cast into the following form

$$
\frac{1}{\bar{P}_{B}} \frac{\partial P_{B}^{2}}{\partial t}-2 P_{B} \frac{\partial \ln T_{B}}{\partial t}=\frac{k_{B}}{\mu}\left[\frac{\partial^{2} P_{B}^{2}}{\partial z^{2}}-\left(\frac{\partial P_{B}^{2}}{\partial z}\right)\left(\frac{\partial \ln T_{B}}{\partial z}\right)\right]+\frac{4}{R_{B}^{2}} \frac{k_{C}}{\mu}\left(\frac{T_{B}}{T_{C}+T_{B}}\right)\left(P_{C}^{2}-P_{B}^{2}\right)
$$

Let us now focus on the energy balance equation in the same elementary volume inside the borehole. The energy balance equation can be expressed as

$$
\left.\pi R_{B}^{2} \Delta z \rho_{B} u_{B}\right|_{t+\Delta t}-\left.\pi R_{B}^{2} \Delta z \rho_{B} u_{B}\right|_{t}=\Delta t\left|\pi R_{B}^{2} q_{B} \rho_{B} h_{B}\right|_{z}-\left.2 \pi R_{B}^{2} q_{B} \rho_{B} h_{B}\right|_{z+\Delta z}+2 \pi R_{B} \Delta z q_{C B} \rho_{C B} h_{C B} \mid
$$

where $u_{B}$ is the specific internal energy and $h_{B}$ is the specific enthalpy inside the borehole. Further, $h_{C B}$ is the specific enthalpy of the air entering into the borehole from the surrounding continuum. Equation A.7 can be cast into partial differential form as

$$
\frac{\partial \rho_{B} u_{B}}{\partial t}=-\frac{\partial}{\partial z}\left(q_{B} \rho_{B} h_{B}\right)+\frac{2}{R_{B}}\left(q_{C B} \rho_{C B} h_{C B}\right) .
$$

Since ideal gas behavior has been assumed, we introduce $u_{B}=C_{V} T_{B}, h_{B}=C_{P} T_{B}$, and $h_{C B}=C_{P}\left(\frac{T_{C}+T_{B}}{2}\right)$, where $C_{V}$ and $C_{P}$ are the specific heat capacities of an ideal gas under constant volume and constant pressure, respectively. From the properties of an ideal gas, we get $C_{P}-C_{V}=R$. Using these definitions, and using Equations A.3a and A.3b, we obtain (from Equation A.8)

$$
\frac{C_{V}}{C_{P}} \frac{\partial P_{B}}{\partial t}=\frac{k_{B}}{\mu} \frac{\partial}{\partial z}\left(P_{B} \frac{\partial P_{B}}{\partial z}\right)+\frac{1}{R_{B}^{2}} \frac{k_{C}}{\mu}\left(P_{C}^{2}-P_{B}^{2}\right)
$$

Assuming again that properties can be evaluated at some average pressure $\bar{P}_{B}$, we write Equation A.9 as

$$
\frac{1}{\gamma \bar{P}_{B}} \frac{\partial P_{B}^{2}}{\partial t}=\frac{k_{B}}{\mu} \frac{\partial^{2} P_{B}^{2}}{\partial z^{2}}+\frac{2}{R_{B}^{2}} \frac{k_{C}}{\mu}\left(P_{C}^{2}-P_{B}^{2}\right) .
$$

Note that Equation A.10 is a partial differential equation in one unknown variable and can be solved for $P_{B}$.

We now combine Equations A.6 and A.10, and after some algebraic manipulations, we obtain 
$-\frac{(\gamma-1)}{\gamma \bar{P}_{B}} \frac{\partial P_{B}^{2}}{\partial t}+2 P_{B} \frac{\partial \ln T_{B}}{\partial t}=\frac{2}{R_{B}^{2}} \frac{k_{C}}{\mu}\left(P_{C}^{2}-P_{B}^{2}\left[\frac{T_{C}-T_{B}}{T_{C}+T_{B}}\right]+\frac{k_{B}}{\mu}\left(\frac{\partial P_{B}^{2}}{\partial z}\right)\left(\frac{\partial \ln T_{B}}{\partial z}\right)\right.$

Instead of working with $T_{B}$, it is more convenient to make the transformation

$$
\sigma_{B}=\ln \frac{T_{B}}{T_{C}} .
$$

With this transformation of variable, we can write

$$
\frac{T_{C}-T_{B}}{T_{C}+T_{B}}=\frac{1-e^{\sigma_{B}}}{1+e^{\sigma_{B}}}
$$

Using these transformations, we finally obtain

$$
-\frac{(\gamma-1)}{\gamma \bar{P}_{B}} \frac{\partial P_{B}^{2}}{\partial t}+2 P_{B} \frac{\partial \sigma_{B}}{\partial t}=\frac{2}{R_{B}^{2}} \frac{k_{C}}{\mu}\left(P_{C}^{2}-P_{B}^{2}\left[\frac{1-e^{\sigma_{B}}}{1+e^{\sigma_{B}}}\right]+\frac{k_{B}}{\mu}\left(\frac{\partial P_{B}^{2}}{\partial z}\right)\left(\frac{\partial \sigma_{B}}{\partial z}\right)\right.
$$

Since the magnitude of actual temperature drop is relatively small (i.e., $\frac{T_{B}}{T_{C}} \approx 1.0$ ), $\sigma_{B} \approx 0$. Under these circumstances, Equation A.14 can be further simplified as

$$
-\frac{(\gamma-1)}{\gamma \bar{P}_{B}} \frac{\partial P_{B}^{2}}{\partial t}+2 P_{B} \frac{\partial \sigma_{B}}{\partial t}=-\frac{2}{R_{B}^{2}} \frac{k_{C}}{\mu}\left(P_{C}^{2}-P_{B}^{2}\right)\left(\frac{\sigma_{B}}{2}\right)+\frac{k_{B}}{\mu}\left(\frac{\partial P_{B}^{2}}{\partial z}\right)\left(\frac{\partial \sigma_{B}}{\partial z}\right)
$$

Equations A.10 and A.15 can now be solved for the two variables $P_{B}$ and $\sigma_{B}$.

\section{Appendix B}

Before solving the differential equations, we introduce the dimensionless variables $\zeta=\frac{Z}{H}, \tau=\frac{\alpha_{B} t}{H^{2}}$, and $\psi_{B}=\frac{P_{C}^{2}-P_{B}^{2}}{P_{C i}^{2}}$, where $\alpha_{B}=\frac{\not k_{B} \bar{P}_{B}}{\mu}$. After transforming to these dimensionless variables, Equation 2 becomes

$$
\frac{\partial \psi_{B}}{\partial \tau}=\frac{\partial^{2} \psi_{B}}{\partial \zeta^{2}}-\frac{2 H^{2}}{R_{B}^{2}}\left(\frac{k_{C}}{k_{B}}\right) \psi_{B}
$$


Since we have defined $\varepsilon_{C B}=\frac{2 H^{2}}{R_{B}^{2}}\left(\frac{k_{C}}{k_{B}}\right)$ as representing an interaction parameter between the surrounding continuum and the borehole, Equation B.1 can be rewritten as

$$
\frac{\partial \psi_{B}}{\partial \tau}=\frac{\partial^{2} \psi_{B}}{\partial \zeta^{2}}-\varepsilon_{C B} \psi_{B}
$$

The initial and boundary conditions for Equation B.2 are

$$
\psi_{B}(\zeta, 0)=0
$$

$$
\left.\frac{\partial \psi_{B}}{\partial \zeta}\right|_{\zeta=0}=0
$$

and

$$
\left.\frac{\partial \psi_{B}}{\partial \zeta}\right|_{\zeta=1}=\dot{m}_{D}
$$

where $\dot{m}_{D}=\frac{\dot{m}}{\pi R_{B}^{2}\left(\frac{k_{B} P_{C}}{\mu H}\right)\left(\frac{P_{C} M_{a}}{2 R T_{C}}\right)}$.

Subject to these initial and boundary conditions, Equation B.2 can be solved in Laplace transform space as

$$
\bar{\psi}_{B}(\zeta, s)=\dot{m}_{D} \frac{\cosh \zeta \sqrt{s+\varepsilon_{C B}}}{s \sqrt{s+\varepsilon_{C B}} \sinh \sqrt{s+\varepsilon_{C B}}},
$$

where $s$ is the Laplace transform parameter in the transformation

$$
\bar{\psi}_{B}(\zeta, s)=\int_{0}^{\infty} \psi_{B}(\zeta, \tau) e^{-s \tau} d \tau
$$

Equation B.4 can be readily inverted back into real time space to yield [Abramowitz and Stegun, 1964] 


$$
\psi_{B}(\zeta, \tau)=\dot{m}_{D}\left[\frac{1}{\varepsilon_{C B}}\left(1-e^{-\varepsilon_{C B} \tau}\right)+2 \sum_{n=1}^{\infty} \frac{(-1)^{n}}{n^{2} \pi^{2}+\varepsilon_{C B}}\left\{1-e^{-\left(n^{2} \pi^{2}+\varepsilon_{C B}\right) \tau}\right\} \cos n \pi \zeta\right]
$$

Having obtained an expression for the dimensionless pressure drop in the borehole, we now turn our attention to solving Equation A.15, to obtain a solution for reduced temperature $\sigma_{B}$. Casting Equation A.15 into dimensionless form, we obtain, after some manipulations

$$
(\gamma-1) \frac{\partial \psi_{B}}{\partial \tau}+2 \gamma\left(1-\psi_{B}\right) \frac{\partial \sigma_{B}}{\partial \tau}=-\varepsilon_{C B} \psi_{B}\left(\frac{\sigma_{B}}{2}\right)-\left(\frac{\partial \psi_{B}}{\partial \zeta}\right)\left(\frac{\partial \sigma_{B}}{\partial \zeta}\right)
$$

To further simplify, we introduce a new variable

$$
y_{B}=\ln \left(1-\psi_{B}\right)
$$

With this transformation, Equation B.6 reduces to

$$
-(\gamma-1) \frac{\partial y_{B}}{\partial \tau}+2 \gamma \frac{\partial \sigma_{B}}{\partial \tau}=\varepsilon_{C B}\left(\frac{\sigma_{B}}{2}\right)\left(1-e^{-y_{B}}\right)+\left(\frac{\partial y_{B}}{\partial \zeta}\right)\left(\frac{\partial \sigma_{B}}{\partial \zeta}\right)
$$

Equation B.8 is a first-order partial differential equation in $\sigma_{B}\left(y_{B}\right.$ is already known) and needs to be solved for $\sigma_{B}$. The initial and boundary conditions are

$$
\begin{gathered}
\sigma_{B}(\zeta, 0)=0 \\
\sigma_{B}\left(\zeta_{F}, \tau\right)=0
\end{gathered}
$$

where $\zeta_{F}=(h+b) / H$ is the dimensionless location of the fracture. Because of the nonlinear nature of Equation B.8, an analytical solution cannot be found directly. However, an implicit finite difference solution can be easily obtained (subject to the constraints in B.9a and B.9b).

\section{Appendix C}

The mass rate of (air) flow into the borehole can be expressed as 
$\dot{m}_{i n}=\frac{2 \pi R_{B} k_{C}}{\mu} \int_{0}^{h} \rho\left(\frac{P_{C}-P_{B}}{R_{B}}\right) d z+\frac{2 \pi R_{B} k_{C}}{\mu} \int_{h+2 b}^{H} \rho\left(\frac{P_{C}-P_{B}}{R_{B}}\right) d z+\frac{2 \pi R_{B} k_{F}}{\mu} \rho(2 b)\left(\frac{P_{C}-P_{B}}{R_{B}}\right)$

While the first term on the right hand side of Equation (C.1) can be considered the contribution to overall air flow rate from the fracture continuum below the discrete fracture (the discrete fracture is located $h \mathrm{~m}$ above the bottom of the borehole, the end opposite to the end where pumping is applied), the second term is the contribution from that above the discrete fracture (the overall length of the packed-off section is $H \mathrm{~m}$ ). The third term, on the other hand, is the contribution to the overall flow rate from the flow in the discrete fracture.

Since (2b), the thickness of the discrete fracture (of the order of 10 to 100 microns), is significantly smaller than the other dimensions ( $h$ or $H$ ), the third term is negligible (even if $k_{F}$ is two orders of magnitude larger than $k_{C}$ ) compared to the first and second terms in Equation C.1. Thus for small values of (2b), Equation C.1 can be approximately written as

$$
\dot{m}_{i n}=\int_{0}^{H} 2 \pi R_{B} \rho \frac{k_{C}}{\mu}\left(\frac{P_{C}-P_{B}}{R_{B}}\right) d z
$$

The density of air coming into the borehole can be approximated (assuming ideal gas law) as

$$
\rho=\frac{M_{a}}{R}\left(\frac{P_{C}+P_{B}}{T_{C}+T_{B}}\right)
$$

Under these conditions, Equation C.2 becomes, assuming that the viscosity of air remain constant,

$$
\dot{m}_{i n}=2 \pi R_{B} \frac{k_{C}}{\mu}\left(\frac{M_{a}}{R R_{B}}\right) \int_{0}^{H} \frac{P_{C}^{2}-P_{B}^{2}}{T_{C}+T_{B}} d z
$$

Introducing the dimensionless variables discussed in Appendix B, Equation C.4 can be reduced to 


$$
\dot{m}_{i n}=2\left(\frac{H^{2}}{R_{B}^{2}}\right)\left(\frac{k_{C}}{k_{B}}\right)\left[\pi R_{B}^{2}\left(\frac{K_{B} P_{C}}{\mu H}\right)\left(\frac{P_{C} M_{a}}{R T_{C}}\right)\right] \int_{0}^{1} \frac{2 \psi_{B}}{1+\exp \left(\sigma_{B}\right)} d \zeta
$$

Notice that for the changes in temperature observed in a typical fluid logging experiment $\sigma_{B} \approx 0$ (i.e., $T_{B} / T_{C} \approx 1$ ). Using this observation, and utilizing the definitions of $\dot{m}_{D}$ (from Equation 5) and $\varepsilon_{C B}$ (from Equation 6), we can write Equation C.5 as

$$
\dot{m}_{i n}=\varepsilon_{C B} \frac{\dot{m}}{\dot{m}_{D}} \int_{0}^{1} \psi_{B} d \zeta
$$

Inserting the expression for $\psi_{B}$ (from Equation 4) into Equation 5 and after performing the required integration, we finally obtain

$$
\dot{m}_{i n}=\dot{m}\left[1-\exp \left(-\varepsilon_{C B} \tau\right)\right]
$$

\section{Figure Captions}

Figure 1a. Photograph of the whole FFTL tool (from Tsang et al., 2007)

Figure 1b. Close-up view of the FFTL tool (from Tsang et al., 2007)

Figure 2a. Sample FFTL pressure and pumping data from Borehole 182 of the ESF at Yucca Mountain, Nevada

Figure 2b. FFTL temperature data from the logging experiment in Figure 2a

Figure 3. Schematic representation of the conceptual model for a FFTL experiment

Figure 4a. Comparison of analytical results and TOUGH2 simulations of a singlephase synthetic FFTL experiment at small times (less than 5 seconds)

Figure 4b. Comparison of analytical results and TOUGH2 simulations of a singlephase synthetic FFTL experiment at large times (larger than 5 seconds)

Figure 5. Pressure and temperature response from a FFTL experiment for various values of the parameter $\varepsilon_{C B}$

Figure 6a. Detection of a discrete fracture from temperature response of a FFTL: comparison of temperature profile at different times when $\varepsilon_{C B}=0.005$ 
Figure 6b. Detection of a discrete fracture from temperature response of a FFTL: comparison of temperature profile for different values of the parameter $\varepsilon_{C B}$ when time is 10.2 seconds

Figure 7. Temperature (both analytical and TOUGH2 predictions), and ratio of inflow and pumping rates from a synthetic FFTL experiment. The figure also shows $t_{c}$ (critical time) at which temperature begins its upward climb. $t_{c}$ can be used to estimate the effective permeability of the fracture continuum

\section{Table Caption}

Table 1. List of parameters and their values used in generating the synthetic FFTL pressure and temperature data

Table 1.

\begin{tabular}{|l|l|l|}
\hline Parameter Name & Symbol & Value \\
\hline Borehole radius & $R_{B}$ & $0.04 \mathrm{~m}$ \\
\hline Length of packed-off zone & $H$ & $2.0 \mathrm{~m}$ \\
\hline Borehole permeability & $k_{B}$ & $10^{-8} \mathrm{~m}^{2}$ \\
\hline Discrete fracture permeability & $k_{F}$ & $10^{-10} \mathrm{~m}^{2}$ \\
\hline Fracture continuum permeability & $k_{C}$ & $10^{-12} \mathrm{~m}^{2}$ \\
\hline Air viscosity & $\mu$ & $1.86 \times 10^{-5} \mathrm{~kg} \mathrm{~m}^{-1} \mathrm{~s}^{-1}$ \\
\hline Fracture continuum pressure & $P_{C}$ & $1 \times 10^{5} \mathrm{~Pa}$ \\
\hline Fracture continuum temperature & $T_{C}$ & $24^{\circ} \mathrm{C}$ \\
\hline Initial pressure & $P_{i}$ & $1 \times 10^{5} \mathrm{~Pa}$ \\
\hline Initial temperature & $T_{i}$ & $24^{\circ} \mathrm{C}$ \\
\hline Pumping rate & $\dot{m}$ & $5.5 \times 10^{-4} \mathrm{~kg} \mathrm{~s}^{-1}$ \\
\hline
\end{tabular}



a

b 
$a$

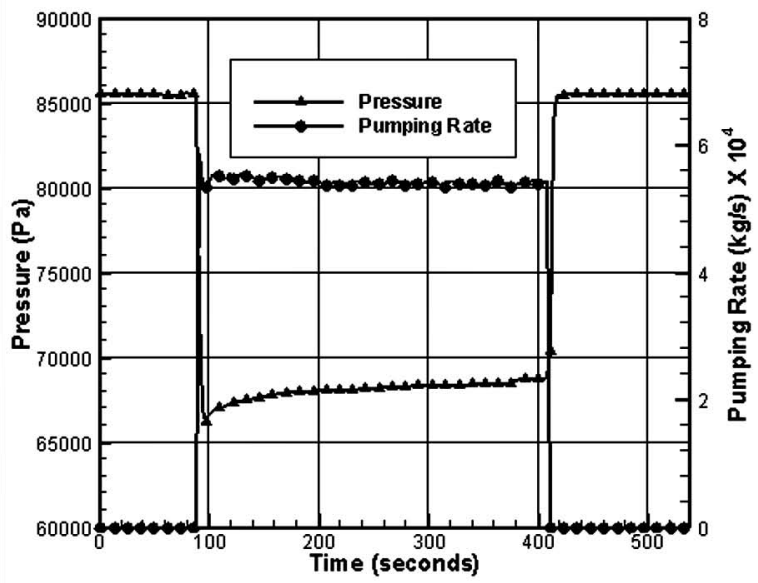

b

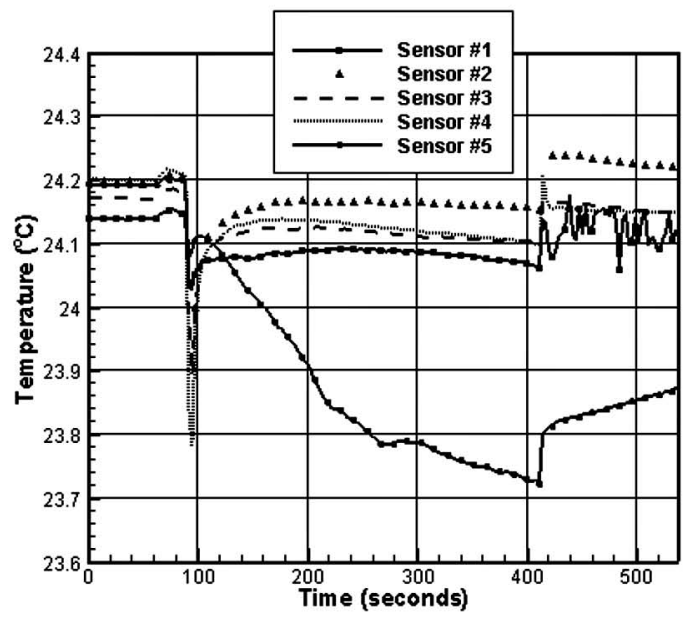




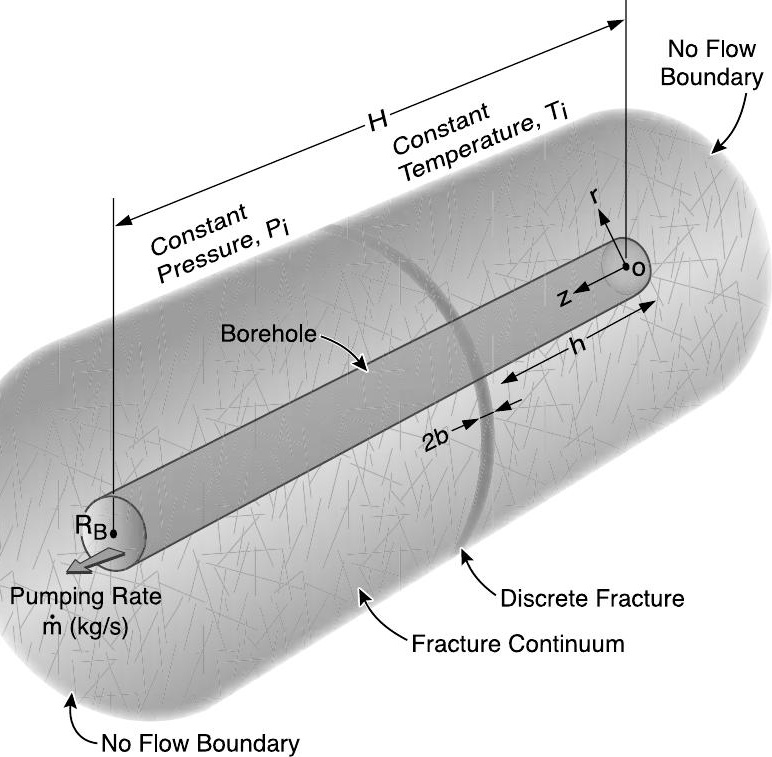



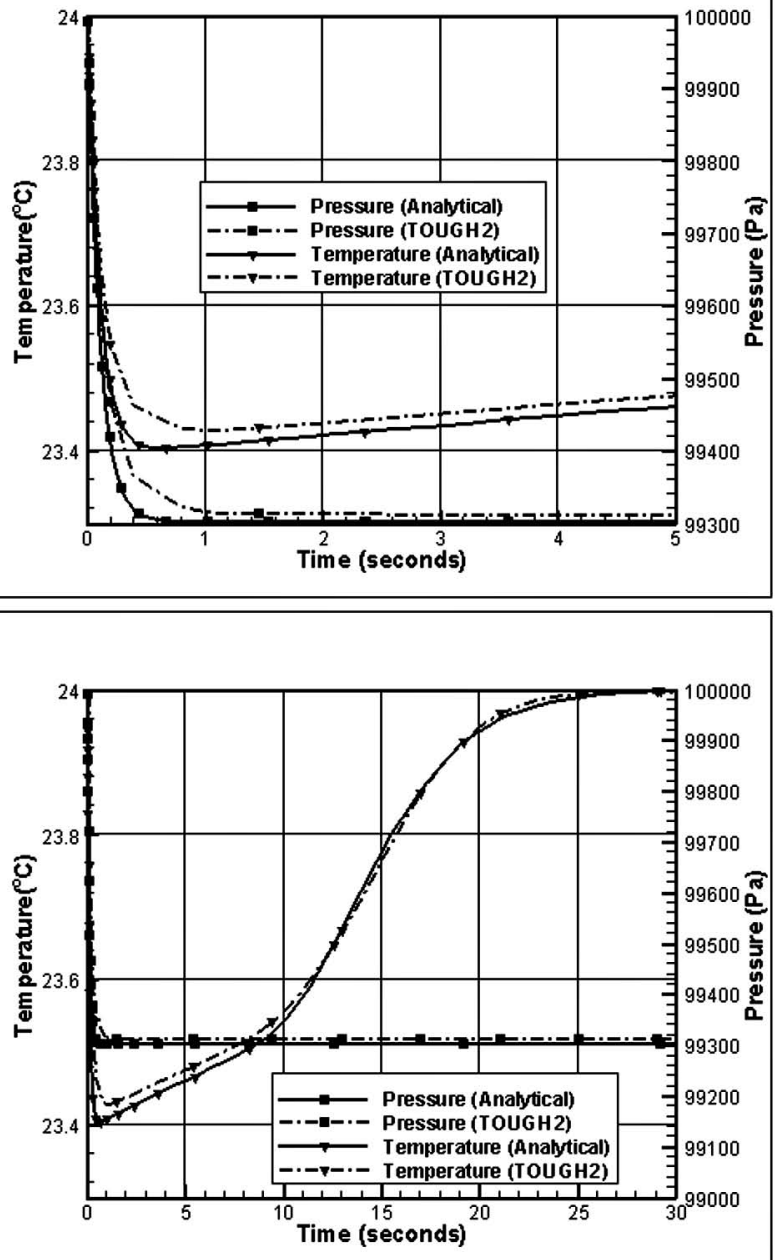


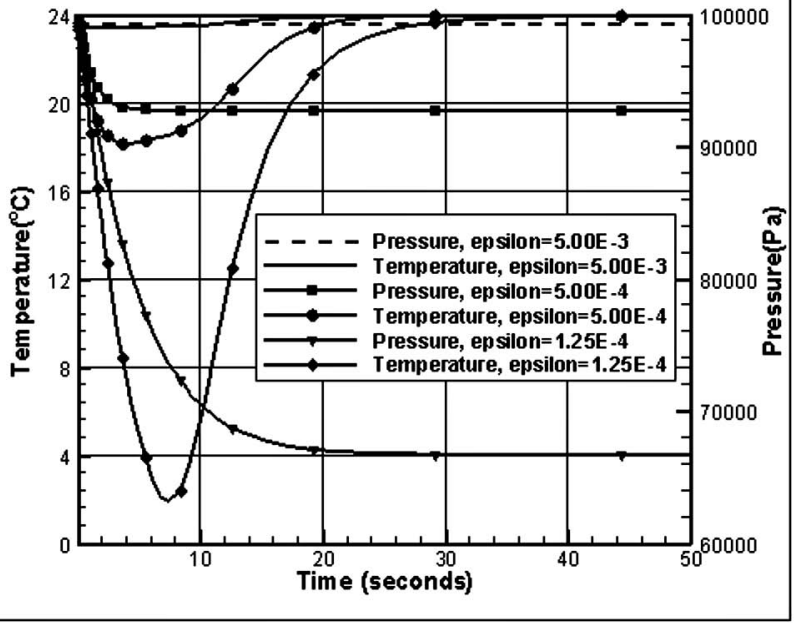




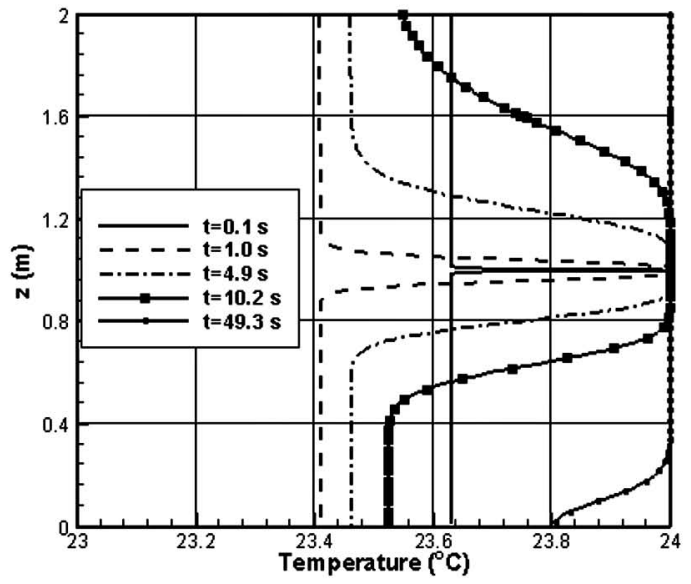

b

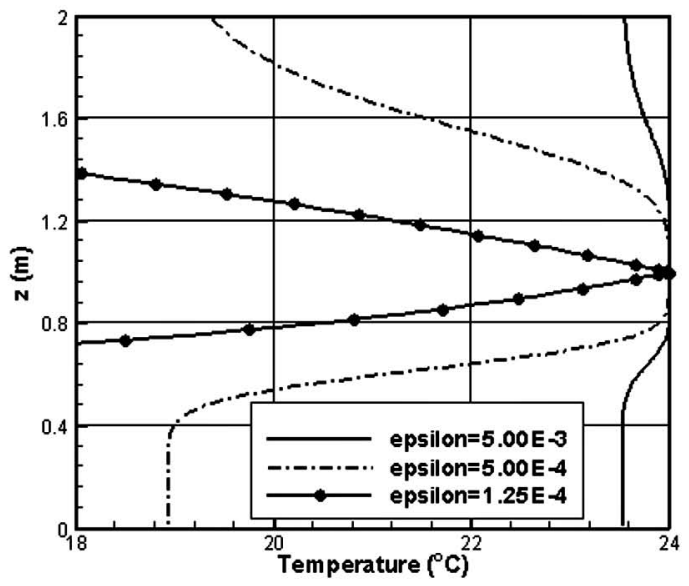




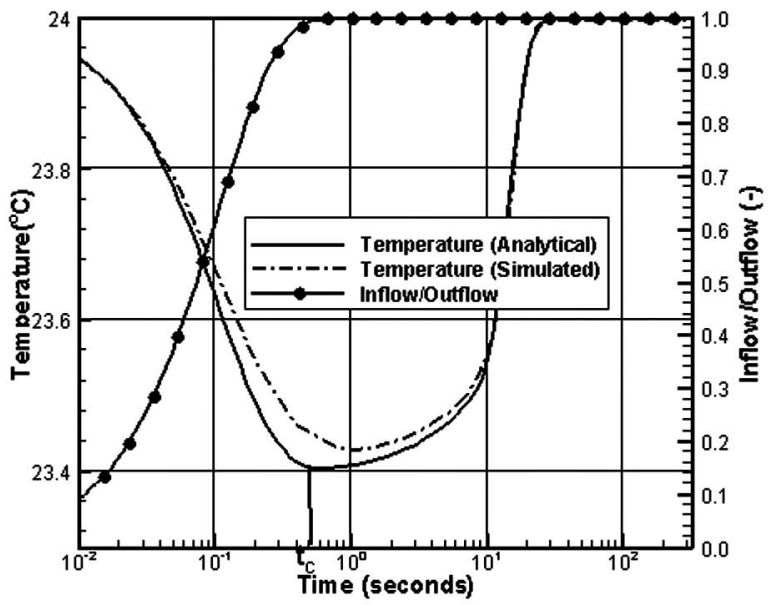

